GUM services, were younger (mean age: 30.5 yrs vs 38.5 yrs; $\mathrm{p}<0.001)$, and more likely to be of black ethnicity $(6.8 \%$ vs $4.1 \% ; \mathrm{p}<0.001)$ and reside in London $(49.9 \%$ vs $46.0 \%$; $\mathrm{p}<0.001)$. MSM attending non-GUM services were more likely to have a full sexual health screen $(41.4 \%$ vs $27.0 \%$; p < $0.001)$, HIV test $(8.9 \%$ vs $7.1 \%$; $<<0.001)$, and be diagnosed with chlamydia $(6.2 \%$ vs $3.0 \%$; $p<0.001)$, gonorrhoea $(5.6 \%$ vs $4.6 \%: \mathrm{p}<0.001)$ and first-episode genital warts $(1.5 \%$ vs 1.0\%: $\mathrm{p}<0.001)$. There was no significant difference in the proportion newly diagnosed with HIV $(0.57 \%$ vs $0.69 \%$; $\mathrm{p}=0.268)$ or first-episode genital herpes $(0.47 \%$ vs $0.46 \%$; $\mathrm{p}=0.830)$.

Conclusion Level 2 sexual health services play an important role in the sexual health care of MSM, especially those of younger age.

\section{P97 MISSED OPPORTUNITIES FOR DIAGNOSING HIV IN A DISTRICT GENERAL HOSPITAL IN AN AREA OF HIGH HIV PREVALENCE}

Matthew James*, Eleanor Draeger, Charles Mazhude, Ruhin Das, Melanie Rosenvinge. University Hospital Lewisham, London, UK

\subsection{6/sextrans-2015-052126.140}

Background Delayed diagnosis of HIV is associated with significantly increased morbidity and mortality. Our clinic has a high rate of advanced HIV at diagnosis $(61 \%$ presenting with a CD4 $<350$ ) indicating that there may be missed opportunities for earlier testing.

Aim To review all recent new diagnoses of HIV for potential missed testing opportunities.

Methods Retrospective review of clinic, hospital and emergency department records for all new patients referred to the HIV clinic between January 2014 and January 2015. Previous hospital admissions, outpatient and emergency department attendances and GP visits were reviewed for the year up to diagnosis. Where a patient was admitted to hospital, time to diagnosis, outcome and inpatient stay was recorded.

Results 70 new patients: 24 transfers of care (excluded); 46 new diagnoses.

\begin{tabular}{llll}
\hline Gender: female & $18 / 46(39 \%)$ & CD4 Count & $\begin{array}{l}29 / 46(63 \%) \text { CD4 <350 } \\
11 / 46(24 \%) \text { CD4 <100 } \\
\text { Mean CD4 Count 322 } \\
\text { Sexuality: MSM }\end{array}$ \\
& $17 / 46(37 \%)$ & Referral Route & $\begin{array}{l}\text { SRH 13/46 (28\%) } \\
\text { Inpatient 10/46 (22\%) } \\
\text { GP 10/46 (22\%) }\end{array}$ \\
& & Other 13/46 (28\%) \\
Country of birth & & \\
UK & $12 / 45(27 \%)$ & \\
Sub-Saharan Africa & $23 / 45(51 \%)$ & \\
Other & $10 / 45(22 \%)$ & \\
\hline
\end{tabular}

$24 / 46(52 \%)$ were seen at least once at the hospital or by the GP in the 12 months prior to their diagnosis. 14 admissions to hospital at the time of diagnosis: mean length of stay 14 days (range 2-47).

Discussion There are significant opportunities for earlier HIV testing in our hospital and local GP practices. We are using this data as part of a business case to roll out HIV testing for all acute medical admissions.

\section{P98 HIV TESTING IN SOUTH LONDON}

Sharenja Ratnakumar*, Melanie Rosenvinge, Thembi Moyo, Tina Ibe, Faith Odong. University Hospital Lewisham, London, UK

\subsection{6/sextrans-2015-052126.141}

Background Early diagnosis is an important factor associated with HIV-related mortality, morbidity and onward transmission. The local prevalence is estimated at 7.8 per 1000 population and $61 \%$ of patients are diagnosed with a CD4 count of $<350$. Despite the National HIV testing guidelines being published in 2008, local HIV testing remains low due to lack of resources, funding and clinical awareness.

Objective To pilot routine HIV testing of all medical admissions during National HIV testing week.

Methods General medical admissions during $22^{\text {nd }}-30^{\text {th }}$ November 2014 were offered a third generation INSTI HIV point of care test (POCT) the morning after admission. A\&E attendees between 9 am and $4 \mathrm{pm}$ on $1^{\text {st }}$ December 2014 (World AIDS day) were also offered POCTs. Basic demographics were collected and analysed with appropriate statistical tests.

Results 141 POCTs were offered in medical admissions; all 126 individuals who accepted (89\%) tested negative (64 white British (51\%), 10 black African (8\%)). 14 refused testing; 9 tested before. 21 individuals were not offered POCTs due to unavailability/ inappropriateness. There was no statistical difference in mean ages or proportion of females/males that accepted or refused testing in this group. 32 patients tested in A\&E were all negative (11 black African (34\%)).

\begin{tabular}{|c|c|c|c|}
\hline Category & Medical & A\&E & Two tailed $\mathrm{P}$ values ${ }^{*}$ t-test, ${ }^{*}$ ZZ-ratio \\
\hline \multirow[t]{2}{*}{$\overline{\text { Age }}$} & 56.9 & 41.6 & ${ }^{*} \mathrm{P}<0.0001$ \\
\hline & $(n=126)$ & $(n=32)$ & \\
\hline \multicolumn{4}{|l|}{ Ethnicity } \\
\hline Black African & $n=1$ & $\mathrm{n}=11$ & ${ }^{* *} \mathrm{P}<0.0002$ \\
\hline White British & $\mathrm{n}=64$ & $n=8$ & ${ }^{* *} \mathrm{P}<0.0089$ \\
\hline
\end{tabular}

Discussion There was a high uptake of HIV testing amongst general medical admissions indicating routine testing is very acceptable to patients. Moreover, a younger population group presents in A\&E compared to admissions; a significant proportion being Black African origin. This may be an appropriate target group to consider for testing.

\section{P99 RENAL AND BONE SAFETY OF TENOFOVIR ALAFENAMIDE VS TENOFOVIR DISOPROXIL FUMARATE}

${ }^{1}$ Frank Post*, ${ }^{2}$ Paul Sax, ${ }^{3}$ Michael Saag, ${ }^{4}$ Michael Yin, ${ }^{5}$ Shinichi Oka, ${ }^{6}$ Ellen Koenig, ${ }^{7}$ Benoit Trottier, ${ }^{8}$ Jaime Andrade, ${ }^{9}$ Huyen Cao, ${ }^{9}$ Marshall Fordyce. ${ }^{1}$ King's College Hospital, London, UK; ${ }^{2}$ Brigham and Women's Hospital, Boston, UK; ${ }^{3}$ University of Alabama, Alabama, USA; ${ }^{4}$ Columbia University, New York, USA; ${ }^{5}$ International Medical Centre of Japan, Tokyo, USA; ${ }^{6}$ Dominican Institute for Virological Studies, Santo Domingo, Japan; ${ }^{7}$ Clinique Medicale L'Actuel in Montreal, Montreal, Dominican Republic; ${ }^{8}$ Hospital Civil de Guadalajara, Guadalajara, Canada; ${ }^{9}$ Gilead Sciences Inc, Foster City, Mexico

\subsection{6/sextrans-2015-052126.142}

Background Off-target renal and bone side effects may occur with tenofovir disoproxil fumarate (TDF) use. Compared with TDF, tenofovir alafenamide (TAF) results in significantly reduced plasma tenofovir (TFV) and may have less renal and bone toxicity. 
Methods Treatment naïve HIV-1+ adults were randomised 1:1 to a single tablet regimen of $\mathrm{E} / \mathrm{C} / \mathrm{F} / \mathrm{TAF}$ or $\mathrm{E} / \mathrm{C} / \mathrm{F} / \mathrm{TDF}$ once daily in two double blind studies. Assessments for all subjects included measures of glomerular and proximal renal tubular function, and bone mineral density (BMD). Four pre-specified secondary safety endpoints were tested: serum creatinine, treatment-emergent proteinuria, spine and hip BMD. Week 48 off-target side effects data are described.

Results 1,733 subjects were randomised and treated. Plasma TFV was $>90 \%$ lower (mean $\mathrm{AUC}_{\text {tau }} 297$ vs. 3,410 ng.hr/mL) in the $\mathrm{E} / \mathrm{C} / \mathrm{F} / \mathrm{TAF}$ arm, compared to the $\mathrm{E} / \mathrm{C} / \mathrm{F} / \mathrm{TDF}$ arm. Serum creatinine (mean change: +0.08 vs $+0.11 \mathrm{mg} / \mathrm{dL}, \mathrm{p}<0.001$ ), quantified proteinuria (UPCR, median \% change; -3 vs +20 , $\mathrm{p}<0.001$ ), and fractional excretion of phosphate (median $\%$ change; +0.9 vs +1.7 ), all favoured E/C/F/TAF. There were no cases of proximal tubulopathy in either arm. Mean $\%$ decrease in BMD was significantly less in the $\mathrm{E} / \mathrm{C} / \mathrm{F} / \mathrm{TAF}$ arm for both lumbar spine $(-1.30$ vs $-2.86, \mathrm{p}<0.001)$ and total hip $(-0.66$ vs $-2.95, \mathrm{p}<0.001)$.

Conclusions Through 48 weeks, subjects receiving E/C/F/TAF had significantly better outcomes related to renal and bone health than those treated with $\mathrm{E} / \mathrm{C} / \mathrm{F} / \mathrm{TDF}$. These data demonstrate important safety benefits of TAF relative to TDF, especially given the ageing of the HIV population and the need for long-term treatment.

\section{P100 TENOFOVIR ALAFENAMIDE (TAF) IN A SINGLE TABLET REGIMEN IN INITIAL HIV-1 THERAPY}

${ }^{1}$ Anton Pozniak*, ${ }^{2}$ David Wohl, ${ }^{3}$ Melanie Thompson, ${ }^{4}$ Edwin Dejesus, ${ }^{5}$ Daniel Podzamczer, ${ }^{6}$ Jean Michel Molina, ${ }^{7}$ Gordon Crofoot, ${ }^{8}$ Christian Callebaut, ${ }^{8}$ Hal Martin, ${ }^{8}$ Scott McCallister. ${ }^{1}$ Chelsea and Westminster NHS Hospital, London, UK; ${ }^{2}$ North Carolina Department of Corrections, North Carolina, Uganda; ${ }^{3}$ AIDS Research Consortium of Atlanta, Atlanta, USA; ${ }^{4}$ Orlando Immunology Center, Orlando, USA; ${ }^{5}$ L'Hospitalet de Llobregat, Barcelona, USA; ${ }^{8}$ Gilead Sciences Inc, Foster City, USA; ${ }^{6}$ Hôpital Saint-Louis, Paris, Spain; ${ }^{7}$ Premier Medical Practice, Houston, France

\subsection{6/sextrans-2015-052126.143}

Background Tenofovir alafenamide (TAF) is a novel tenofovir (TFV) prodrug that, when administered in the single tablet regimen $\mathrm{E} / \mathrm{C} / \mathrm{F} / \mathrm{TAF}$, has $>90 \%$ lower plasma TFV levels compared to tenofovir disoproxil fumarate (TDF).

Methods Treatment naïve HIV-1+ adults were randomised 1:1 to receive a regimen of $\mathrm{E} / \mathrm{C} / \mathrm{F} / \mathrm{TAF}$ or $\mathrm{E} / \mathrm{C} / \mathrm{F} / \mathrm{TDF}$ in two Phase 3 double blind studies. Primary endpoint was Week 48 virologic response by FDA Snapshot algorithm in a pre-specified combined analysis.

Results 1,733 subjects were randomised and treated: 15\% women, $43 \%$ non-White, $23 \%$ viral load $\geq 100,000$ copies $/ \mathrm{mL}$. The primary objective was met, E/C/F/TAF was non-inferior to $\mathrm{E} / \mathrm{C} / \mathrm{F} / \mathrm{TDF}$ with $92 \%$ and $90 \%$, respectively having HIV RNA $<50$ copies $/ \mathrm{mL}$ at week 48 (difference $+2 \%, 95 \%$ CI $-0.7 \%$ to $+4.7 \%, \mathrm{p}=0.13)$. Virologic failure with resistance occurred in $0.8 \%$ in the $\mathrm{E} / \mathrm{C} / \mathrm{F} / \mathrm{TAF}$ arm and $0.6 \%$ on $\mathrm{E} / \mathrm{C} / \mathrm{F} / \mathrm{TDF}$. Treatment related SAEs were rare: E/C/F/TAF $0.3 \%(\mathrm{n}=3), \mathrm{E} / \mathrm{C} / \mathrm{F} / \mathrm{TDF}$ $0.2 \%(\mathrm{n}=2)$. There were no reports of proximal renal tubulopathy in either arm. No single $\mathrm{AE}$ led to discontinuation of more than 1 subject on E/C/F/TAF. Grade 2, to 4 AEs occurring in $\geq$ $2 \%$ were: diarrhoea (3.3\% vs. $2.5 \%)$, nausea (2.2\% vs. $2.0 \%)$, headache $(2.9 \%$ vs. $2.1 \%)$, and URI $(3.6 \%$ vs. $3.1 \%)$ in the $\mathrm{E} / \mathrm{C} /$ $\mathrm{F} / \mathrm{TAF}$ vs. $\mathrm{E} / \mathrm{C} / \mathrm{F} / \mathrm{TDF}$ arms.

Conclusions Through 48 weeks of treatment, high virologic response rates were seen in patients receiving $\mathrm{E} / \mathrm{C} / \mathrm{F} / \mathrm{TAF}$ or $\mathrm{E} / \mathrm{C} /$
F/TDF. Both regimens were well tolerated, and no unique AEs associated with TAF occurred. These data support the use of E/ $\mathrm{C} / \mathrm{F} / \mathrm{TAF}$, as a potential regimen for initial treatment of patients with HIV-1 infection.

\section{P101 HOW SOON ARE PATIENTS TESTING OUTSIDE GUM RECEIVING A POSITIVE HIV RESULT?}

Naa Torshie Annan*, Ramalingam Nadarajah. Frimley Park Hospital NHS Foundation Trust, Surrey, UK

\subsection{6/sextrans-2015-052126.144}

Introduction UK national guidelines for HIV testing 2008, recommend that any individual testing positive for HIV should see a specialist preferably within $48 \mathrm{~h}$ and certainly two weeks of receiving the result.

Methods All HIV positive test results performed outside the GUM clinic between January 2013 and December 2014 were obtained from the microbiology database at Frimley Park Hospital. 35 patients were identified. 20 were excluded because they were previously known to have HIV, had a "non-specific" or "weakly reactive" result.

Results Of the 15 new diagnosis, 8/15 were inpatients, 4/15 outpatients and 3/15 GP diagnosis. Two-thirds were male, 53\% White British and $73 \%$ heterosexual. The average age was 46 (31-65) years. All the patients had a fourth generation HIV test and a confirmatory test. The majority $(87 \%)$ were late diagnosis with symptomatic HIV/AIDS and an average CD4 count of 50 cells $/ \mathrm{mm}^{3}$. One inpatient diagnosis was missed for 5 weeks until the patient re-presented with PCP. The rest were all seen by a specialist (HIV consultant or health advisor) within 2 weeks of receiving their diagnosis with $64 \%$ seen within $24-48 \mathrm{~h}$.

Discussion/conclusion The majority were late, symptomatic patients with AIDS. All but one result which was initially missed were seen by a specialist within the recommended 2 to 14 days after diagnosis. The recommendation now is that all positive results are phoned to the named consultant/GP responsible for the patient as well as the HIV/GUM team.

\section{Category: Improving clinical practice and service delivery}

\section{P102 WHY DO PATIENTS ATTEND AS REBOOK ATTENDEES IN SEXUAL HEALTH CLINICS?}

Sumit Bhaduri* William Spice. Department of Sexual Health, Worcestershire Health and Care NHS Trust, Worcestershire, UK

\subsection{6/sextrans-2015-052126.145}

Background/introduction Locally commissioners have raised concerns as regards the number of patients re-attending as a new episode of care (rebook) at the countywide sexual health clinics, rebook patients accounting for 54\% of new/rebook attendances over a three month period. Commissioning concerns focussed on whether re attendance for recurrent bacterial sexually transmitted infections (STIs) due to previous suboptimal health prevention/promotion. Are there any grounds for these concerns? Aim(s)/objectives To ascertain the reasons why patients re-attend clinics as rebook patients.

Methods A retrospective analysis of 150 case notes of rebook patients was undertaken with respect to age, gender and reasons 